\title{
VARIATIONS, CHARACTERISTIC CLASSES, AND THE OBSTRUCTION TO MAPPING SMOOTH TO CONTINUOUS COHOMOLOGY
}

\author{
BY
}

MARK A. MOSTOW

\begin{abstract}
In a recent paper, the author gave an example of a singular foliation on $\mathbf{R}^{2}$ for which it is impossible to map the de Rham cohomology $T_{\mathrm{DR}}$ to the continuous singular cohomology $T_{\mathrm{c}}$ (in the sense of Bott and Haefliger's continuous cohomology of spaces with two topologies) compatibly with evaluation of cohomology classes on homology classes. In this paper the obstruction to mapping $T_{\mathrm{DR}}$ to $T_{\mathrm{c}}$ is pinpointed by defining a whole family of cohomology theories $T_{k, m, n}$, based on cochains which vary in a $C^{k}$ manner, which mediate between the two. It is shown that the obstruction vanishes on nonsingularly foliated manifolds. The cohomology theories are extended to Haefliger's classifying space $\left(B \Gamma_{q} \rightarrow B J_{q}\right)$, with its germ and jet topologies, by using a notion of differentiable space similar to those of J. W. Smith and K. T. Chen. The author proposes that certain of the $T_{k m n}$ be used instead of $T_{c}$ to study Bott and Haefliger's conjecture that the continuous cohomology of $\left(B \Gamma_{q} \rightarrow B J_{q}\right)$ equals the relative Gel'fandFuks cohomology $H^{*}\left(a_{q}, O_{q}\right)$. It is shown that $T_{k m n}\left(B \Gamma_{q} \rightarrow B J_{q}\right)$ may contain new characteristic classes for foliations which vary only in a $C^{k}$ manner when a foliation is varied smoothly.
\end{abstract}

0. Introduction. In proving the de Rham isomorphism theorem $H_{\mathrm{DR}}^{*}(M) \cong$ $H_{\text {sing }}^{*}(M ; \mathbf{R})$ for smooth manifolds $M$, one must use two maps to construct the isomorphism. Let $H_{\mathrm{sm}}^{*}$ denote singular cohomology based on smooth simplices. There are maps

$$
H_{\mathrm{DR}}^{*}(M) \stackrel{\alpha}{\rightarrow} H_{\mathrm{sm}}^{*}(M) \stackrel{\beta}{\leftarrow} H_{\text {sing }}^{*}(M) ;
$$

here $\alpha$ is induced by integration of forms over smooth simplices, and $\beta$ by restricting cochains defined on all simplices to the smooth simplices. Part of any proof of the de Rham isomorphism theorem consists of showing that $\beta$ is

Presented to the Society, May 6, 1976 under the title The obstruction to mapping smooth to continuous cohomology of spaces with two topologies; received by the editors May 11, 1976 and, in revised form, November 1, 1976.

AMS (MOS) subject classifications (1970). Primary 55B10, 55B35, 55F40; Secondary 18F05, $18 \mathrm{~F} 15,55-00,55 \mathrm{~B} 30,55 \mathrm{~B} 40,55 \mathrm{G} 35,55 \mathrm{~J} 10,58 \mathrm{~B} 10$.

Key words and phrases. Bott-Haefliger continuous cohomology, space with two topologies, smooth cohomology, foliation, Haefliger classifying space, differentiable space, de Rham theorem, integration of forms, variation of simplices, category of morphisms, geometric realization, simplicial space, Milnor classifying space, variation of characteristic classes, characteristic classes of foliations. 
an isomorphism (see [17, p. 205], [6], [9], for example).

Now an analogous problem arises in the context of continuous cohomology of spaces with two topologies, as defined originally by R. Bott and A. Haefliger in [1] and studied by the author in [11]. We work now in the category $\mathcal{C}_{2}^{\infty}$ whose objects are smooth maps $\left(i: M^{\prime} \rightarrow M\right)$ (also denoted $\mathbf{M}$ ) of smooth manifolds, and whose morphisms $\mathbf{f}: \mathbf{M} \rightarrow \mathbf{N}$ are commutative squares of smooth maps:

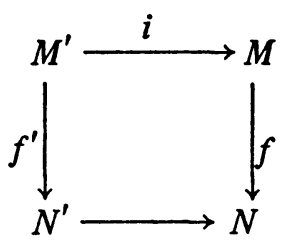

An example of an object in $C_{2}^{\infty}$ arises when $M$ is a manifold with a foliation on it, and $M^{\prime}$ is the set $M$ with the leaf topology, which is defined by making each leaf an open connected component of $M^{\prime}$ and topologizing the leaves as manifolds, not necessarily as subspaces of $M$.

One has a de Rham cohomology theory $T_{\mathrm{DR}}^{*}(\mathbf{M})$ based on differential forms on $M^{\prime}$ which are pullbacks of forms on $M$, and a continuous cohomology theory $T_{c}^{*}(\mathbf{M})$ based on singular real-valued cochains on $M^{\prime}$ which vary continuously as a simplex is moved continuously in $M$. (See below for details. Special cases of $T_{\mathrm{DR}}$ include the homology of the relative de Rham complex of a fibration $\left(M^{\prime} \rightarrow M\right)$ and certain constructions of Kamber and Tondeur (the term $E_{1}^{0, q}(\Omega)$ of $\left[9^{\prime}\right.$, p. 71]) and of Vaisman [16] on foliated manifolds (see also [11, \$4]).)

Simple examples show that $T_{\mathrm{DR}}$ and $T_{\mathrm{c}}$ are not isomorphic, in general. For example, if $M_{d}$ is $M$ with the discrete topology, and $\mathbf{M}_{D}=\left(M_{d} \rightarrow M\right)$, then $T_{\mathrm{DR}}^{0}\left(\mathbf{M}_{D}\right)=C^{\infty}(M)$, while $T_{\mathrm{c}}^{0}\left(\mathrm{M}_{D}\right)=C^{0}(M)$. (Here $C^{k}(M)=\left\{C^{k}\right.$ functions: $M \rightarrow \mathbf{R}\}$.) Nonetheless, one might expect there to exist a welldefined map from $T_{\mathrm{DR}}$ to $T_{\mathrm{c}}$, induced by integrating differential forms over smooth simplices of $M^{\prime}$. Indeed, such a map exists (at the cohomology level) if $M^{\prime}$ is $M$ with the leaf topology of a nonsingular foliation (see Theorem 6). But such a map need not exist, in general, as the following example from [11, \$8] shows. Let $M=\mathbf{R}^{2}$ and let $M^{\prime}$ be $\mathbf{R}^{2}$ topologized as the disjoint union of $\{0\}$ and the concentric circles $S_{r}$ around 0 (where $r=$ radius of $S_{r}$ ). Now each element of $T_{\mathrm{DR}}^{1}(\mathrm{M})$ and of $T_{\mathrm{c}}^{1}(\mathrm{M})$ can be evaluated on the counterclockwise generator of $H_{1}\left(S_{r} ; \mathbf{Z}\right)$; the result is some function of $r$. The calculation in [11] shows that any such function arising from an element of $T_{\mathrm{c}}^{1}(\mathbf{M})$ must vanish in some neighborhood of $r=0$. This is not true for $T_{\mathrm{DR}}^{1}(\mathrm{M})$, however; for example, the differential form $x d y-y d x=r^{2} d \theta$ (a cocycle on $M^{\prime}$ ) leads to the function $2 \pi r^{2}$, which is nonzero except at $r=0$. It follows that there 
can be no map from $T_{\mathrm{DR}}^{1}(\mathrm{M})$ to $T_{\mathrm{c}}^{1}(\mathrm{M})$ respecting evaluation on $H_{1}\left(M^{\prime}\right)=$ $\bigoplus_{r} H_{1}\left(S_{r}\right)$.

In this paper we ask why a map from $T_{\mathrm{DR}}$ to $T_{\mathrm{c}}$ may fail to exist. We show that one may consider separately the differentiability of the simplices, of the variations, and of the cochains in the theory $T_{\mathrm{c}}$. Doing so, we obtain a whole family of cohomology theories $T_{k m n}$ on $C_{2}^{\infty}$ which bridge the gap between $T_{\mathrm{DR}}$ and $T_{\mathrm{c}}$. Certain of the $T_{k m n}$, namely those based on cochains which vary continuously as a simplex is moved differentiably, satisfy the axioms for continuous cohomology presented in [11] (see $\$ 2$ below) and have the advantage over $T_{\mathrm{c}}$ that $T_{\mathrm{DR}}$ does map to them. We therefore propose that one of these $T_{k m n}$ be used instead of $T_{\mathrm{c}}$ in studying Bott and Haefliger's conjecture [1] that the continuous cohomology of $\left(B \Gamma_{q} \rightarrow B J_{q}\right)$ equals the relative Gel'fand-Fuks cohomology $H_{\mathrm{GF}}^{*}\left(\mathfrak{a}_{q}, O_{q}\right)$. (Here $B \Gamma_{q}$ is Haefliger's classifying space for foliations [8], $B J_{q}$ is a related classifying space using the smooth category $J_{q}$ of $\infty$-jets of local diffeomorphisms of $\mathbf{R}^{q}$, with the $C^{\infty}$ topology, $a_{q}$ is the infinite-dimensional Lie algebra of formal vector fields in $q$ variables, and $O_{q}$ is the orthogonal group.) To show that it makes sense to talk about $T_{\mathrm{DR}}\left(B \Gamma_{q} \rightarrow B J_{q}\right)$ and $T_{k m n}\left(B \Gamma_{q} \rightarrow B J_{q}\right)$, we show in the last section that $T_{\mathrm{DR}}$ and $T_{k m n}$ can be defined on (morphisms of) differentiable spaces, generalizations of manifolds similar to those used in the work of J. Wolfgang Smith [14], K. T. Chen [5], and others, and that $B \Gamma_{q}$ and $B J_{q}$ are differentiable spaces. (More details about the differentiable space structures of these and other classifying spaces will appear in [12].) We conclude with some remarks about smooth and continuous variation of characteristic classes for foliations, and suggest that $T_{0 \infty \infty \infty}\left(B \Gamma_{q} \rightarrow B J_{q}\right)$ may contain new characteristic classes.

1. Definitions of the cohomology theories. We work for now in the category $e_{2}^{\infty}$ of maps of manifolds (see Introduction). Our manifolds will all be Hausdorff, finite dimensional, $C^{\infty}$, and a disjoint union of second countable components (hence paracompact). Later we shall work in the larger category $\mathscr{D}_{2}$ whose objects are morphisms of differentiable spaces.

The smooth cohomology theory $T_{\mathrm{DR}}$ on $\mathrm{C}_{2}^{\infty}$ is defined (as in [11]) by

$$
T_{\mathrm{DR}}^{q}\left(i: X^{\prime} \rightarrow X\right)=H^{q}\left(\text { image }\left(i^{*}: A^{*} X \rightarrow A^{*} X^{\prime}\right)\right) \text {, }
$$

where $A^{*} X$ denotes the real vector space of real-valued $C^{\infty}$ differential forms on $X$.

The continuous cohomology theory $T_{\mathrm{c}}$ is defined as follows (see also [11]). Given $\mathbf{X}=\left(i: X^{\prime} \rightarrow X\right) \in \mathcal{C}_{2}^{\infty}$, a $q$-simplex of $\mathbf{X}$ is just a singular $q$-simplex of $X^{\prime}$, i.e. a continuous map $\sigma: \Delta^{q} \rightarrow X^{\prime}$. A continuous variation of $q$-simplices of $\mathbf{X}$ is a set map $v: W \times \Delta^{q} \rightarrow X^{\prime}$ ( $W$ any topological space) such that

(1) Each restriction $v \mid\{w\} \times \Delta^{q}$ (def) $=\sigma_{w}: \Delta^{q} \rightarrow X^{\prime}$ is continuous, and 
(2) The composition iv: $W \times \Delta^{q} \rightarrow X$ is continuous.

N.B. The map $v$ itself need not be continuous.

The variation $v$ is also denoted $w \mapsto \sigma_{w}$, and is said to be parametrized by $W$. A continuous $q$-cochain on $\mathbf{X}$ is an element $h \in S_{\text {singular }}^{q}\left(X^{\prime}, \mathbf{R}\right)$ (i.e. a real-valued function on the $q$-simplices of $X^{\prime}$ ) such that the composition $w \mapsto h\left(\sigma_{w}\right)$ is continuous for each space $W$ and each continuous variation $w \mapsto \sigma_{w}$. (In other words, a continuous cochain on $\mathbf{X}$ is a cochain $h$ on $X^{\prime}$ with the property that $h(\sigma)$ varies continuously when $\sigma$ is moved continuously in $X$; these motions (variations) of $\sigma$ are parametrized by the spaces $W$.) The real vector space of continuous $q$-cochains on $\mathbf{X}$ is denoted $S_{\mathrm{c}}^{q}(\mathrm{X})$. An unsheafified continuous cohomology theory on $C_{2}^{\infty}$ is defined by $H_{\mathrm{c}}^{q}(\mathrm{X})=$ $H S_{\mathrm{c}}^{*}(\mathrm{X})$. We prefer, however, to work with a sheafified theory $T_{\mathrm{c}}$ which satisfies all four axioms of [11] (see [11, p. 27] for an explanation of the obstacle to proving Axiom 3 for $H_{\mathrm{c}}$ ). The presheaf on $X$ defined by $U \rightarrow$ $S_{\mathrm{c}}^{q}(\mathrm{U})\left(U\right.$ open in $X, \mathcal{Q}=\left(i: i^{-1} U \rightarrow U\right)$ ) generates [3, p. 5] a sheaf on $X$ which we denote $\mathbf{S}_{\mathrm{c}}^{q} \mathbf{X}$. Letting $\Gamma$ denote global sections, we define

$$
T_{\mathrm{c}}^{q}(\mathbf{X})=H^{q} \Gamma \mathrm{S}_{\mathrm{c}}^{*} \mathbf{X} \text {. }
$$

We observe that continuity appears three times in the definition of $T_{c}$, namely, in defining the simplices, the variations, and the cochains. We investigate now what happens if we require each of these to satisfy some differentiability requirement (not necessarily the same for each). We begin with a somewhat nonstandard definition of differentiable simplex.

DEFINITION. A real-valued function on $\Delta^{q}$ is $C^{n}$ if it can be extended to a $C^{n}$ function on a neighborhood of $\Delta^{q}$ in $\mathrm{R}^{q}$. A continuous map $\sigma: \Delta^{q} \rightarrow X(X$ a manifold) is a $C^{n}$ simplex of $X$ if it pulls back $C^{n}$ functions on $X$ to $C^{n}$ functions on $\Delta^{q}$.

REMARK. It is more usual (when $X$ is a manifold) to require $\sigma$ to extend to a $C^{n}$ map from a neighborhood of $\Delta^{q}$ to $X$. We use our (equivalent) definition because it will be more adaptable to the case when $X$ is a differentiable space. For example, the identity map of $\Delta^{q}$ is a $C^{n}$ simplex of $\Delta^{q}$ in my definition but does not extend to a $C^{n}$ map from a neighborhood of $\Delta^{q}$ to $\Delta^{q}$.

We define $C^{n}$ maps from $W \times \Delta^{q}$ to $X$ in an analogous way ( $W$ a manifold).

Now let $k, m, n$ each be a nonnegative integer or $\infty$, and let $\mathbf{X} \in C_{2}^{\infty}$.

Definition. A $C^{n} q$-simplex of $\mathbf{X}$ is a $C^{n} q$-simplex of $X^{\prime}$. A $C^{m}$ variation of $C^{n}$ simplices of $\mathbf{X}$ is a set map $v: W \times \Delta^{q} \rightarrow X^{\prime}$ ( $W$ any space if $m=0$, or any manifold if $m>0$ ) such that

(1) each restriction $v \mid\{w\} \times \Delta^{q}=\sigma_{w}: \Delta^{q} \rightarrow X^{\prime}$ is a $C^{n}$ map (simplex).

(2) the composition iv: $W \times \Delta^{q} \rightarrow X$ is $C^{m}$.

A singular cochain $h \in S^{q}\left(X^{\prime}, \mathbf{R}\right)$ is called a $C^{k} q$-cochain on $\mathbf{X}$ with respect to $C^{m}$ variations of $C^{n}$ simplices if for each such variation $w \mapsto \sigma_{w}$, the 
composition $w \mapsto h\left(\sigma_{w}\right)$ is a $C^{k}$ map. The real vector space of all such cochains is denoted $S_{k m n}^{q}(\mathbf{X})$; it is a subspace of $S^{q}\left(X^{\prime} ; \mathbf{R}\right)$.

Remark. One could require $W$ to be a manifold even in the definition of a $C^{0}$ variation; one would get a different theory $T_{\mathrm{c}}$ in this way, but $T_{\mathrm{DR}}$ would still not map to it, as the discussion after Theorem 6 will make clear.

One verifies easily that $S_{k m n}^{q}$ is a contravariant functor from $C_{2}^{\infty}$ to real vector spaces. As before, one defines an unsheafified cohomology theory $H_{k m n}^{q}(\mathbf{X})=H S_{k m n}^{*}(\mathbf{X})$. A sheaf $\mathbf{S}_{k m n}^{q} \mathbf{X}$ on $X$ is generated by the presheaf $U \rightarrow S_{k m n}^{q}(\mathrm{U})$, and a (sheafified) cohomology theory on $\mathrm{C}_{2}^{\infty}$ is defined by

$$
T_{k m n}^{q}(\mathbf{X})=H \Gamma S_{k m n}^{q} \mathbf{X} \text {. }
$$

EXAMPLE. $T_{000}=T_{\mathrm{c}}$.

2. Properties of $T_{\mathrm{DR}}$ and $T_{k m n}$. Our first interest is to see under what conditions on $X^{\prime}, X, k, m$, and $n$ the theories $T_{k m n}$ satisfy the axioms of [11] for continuous or smooth cohomology theories. We recall these axioms now, but present them in a form that includes all $C^{k}$ cohomology theories.

Defintion. Let $\mathcal{C}$ be a subcategory of $\mathcal{C}_{2}^{\infty}$, and let $T^{q}: \mathcal{C} \rightarrow$ Real vector spaces be contravariant functors, $q=0,1,2, \ldots$ Then $T$ is called a $C^{k}$ cohomology theory on $\mathcal{C}$ (satisfying the axioms) if

Axiom 1. $T$ is homotopy invariant in $\mathcal{C}$. That is, if $\mathbf{F}: \mathbf{X} \times \mathbf{R} \rightarrow \mathbf{Y}$ (here $\mathbf{X} \times \mathbf{R}$ means $\left.\left(X^{\prime} \times \mathbf{R} \rightarrow X \times \mathbf{R}\right)\right)$ and $\mathbf{f}_{t}$ denotes $\mathbf{F} \mid \mathbf{X} \times\{t\}, t \in \mathbf{R}$, then

$$
T\left(\mathbf{f}_{0}\right)=T\left(\mathbf{f}_{1}\right): T(\mathbf{Y}) \rightarrow T(X) .
$$

Axıom 2. $T\left(I \mathrm{X}_{i}\right)=\Pi_{i} T\left(\mathrm{X}_{i}\right)$, where $i$ runs over any index set and II is topological sum (disjoint union).

Axiom 3 (Generalized MAYer-Vietoris). Let $\mathcal{Q}=\left\{U_{j}\right\}$ be an open cover of $X$ indexed by a linearly ordered set $J$, let $\vartheta_{p}$ be the topological sum of the $(p+1)$-fold intersections of $\mathcal{U}$, each indexed by a strictly increasing sequence $j_{0}, \ldots, j_{p}$ in $J$, let $Q^{\prime}$ be the pullback of $थ$ to $X^{\prime}$ (via $X^{\prime} \rightarrow X$ ), and let $\partial_{i}: \vartheta_{p+1} \rightarrow \mathcal{U}_{p}(i=0, \ldots, p+1)$ be the "inclusions" induced by omitting the open set indexed by $j_{i}$ in each intersection. Then there is a spectral sequence (natural in $\mathbf{X}$ and $थ$ )

$$
E_{1}^{p, q}=T^{q}\left(\mho_{p}\right) \Rightarrow T^{p+q}(\mathbf{X})
$$

with

$$
d p^{p, q}=\delta=(\text { def }) \sum_{i=0}^{p+1}(-1)^{i} T^{q}\left(\partial_{i}\right): T^{q}\left(\vartheta_{p}\right) \rightarrow T^{q}\left(\vartheta_{p+1}\right)
$$

( $\delta$ is a Čech coboundary operator) provided that the objects $\vartheta_{p}$ and morphisms $T^{q}\left(\partial_{i}\right)$ all belong to $C$.

Remark. If $T$ is defined by $T^{q}=H^{q}\left(S^{*}(\mathbf{X})\right)$ where $\left(S^{*}, d\right)$ is some cochain complex defined naturally for each $\mathbf{X}$, then Axiom 3 is true if the total 
cohomology of the double complex $\bigoplus_{p, q} S^{q}\left(\vartheta_{p}\right)$ under the coboundary $d \pm \delta$ (where $\delta=\Sigma(-1)^{i} S^{q}\left(\partial_{i}\right)$ ) equals $T^{*}(\mathbf{X})$.

Axiom 4 (Normalization). Let $X$ be a $C^{\infty}$ manifold, let $X_{d}$ be $X$ in its discrete topology, and let $\mathbf{X}_{D}=\left(X_{d} \rightarrow X\right)$. Then

$$
T^{q}\left(\mathbf{X}_{D}\right)= \begin{cases}0, & q>0 \\ C^{k}(X), & q=0,\end{cases}
$$

naturally in $X$, where $C^{k}(X)=\left\{C^{k}\right.$ real-valued functions on $\left.X\right\}$.

We recall from [11, Theorems 3.6 and 4.2]

THEOREM 1. $T_{\mathrm{c}}$ is a $C^{0}$ cohomology theory on $C_{2}^{\infty}$ (in fact, also on the larger category $\mathcal{C}_{\text {para }}=\left\{\left(X^{\prime} \rightarrow X\right) \mid X^{\prime}, X\right.$ topological spaces, $X$ paracompact $\left.\}\right)$.

THEOREM 2. $T_{\mathrm{DR}}$ is a $C^{\infty}$ cohomology theory on $\mathrm{C}_{2}^{\infty}$.

By slight modifications of the proof of Theorem 1 (given in [11D we shall prove

THEOREM 3. If $0<k, m, n \leqslant \infty$ and $k<m$ then $T_{k m n}$ is a $C^{k}$ cohomology theory on $\mathcal{C}_{2}^{\infty}$ (satisfying the axioms).

Proof. First observe that if $k<m$, then $S_{k m n}^{q}(X)$ is a module over $C^{\infty}(X)$, via

$$
(f \cdot h)(\sigma)=f(\sigma(0)) \cdot h(\sigma),
$$

where $\sigma: \Delta^{q} \rightarrow X^{\prime}$ is a $C^{n}$ simplex, 0 is any fixed basepoint in $\Delta^{q}, f \in C^{\infty}(X)$, and $h \in S_{k m n}^{q}(\mathbf{X})$. That $f \cdot h$ does in fact lie in $S_{k m n}^{q}(\mathbf{X})$ follows from the fact that if $w \mapsto \sigma_{w}$ is a $C^{m}$ variation of $C^{n}$ simplices, then $w \mapsto i \sigma_{w}(0) \in X$ is $C^{m}$, while $w \mapsto h\left(\sigma_{w}\right)$ is $C^{k}$, so that $w \mapsto(f \cdot h)\left(\sigma_{w}\right)$ is $C^{p}$, where $p=\min (m, k)$.

It follows that $\mathbf{S}_{k m n}^{q} \mathbf{X}$ is a module over the sheaf $C^{\infty} X$ on $X$ of germs of smooth real-valued functions on $X$. Since $\mathbf{C}^{\infty} X$ is a fine sheaf of rings with unit (via smooth partitions of unity) [17, p. 170] and $X$ is paracompact, it follows [3, p. 50] that $\mathbf{S}_{k m n}^{q} \mathbf{X}$ is a fine sheaf.

Axioms 2 and 3 now follow from standard sheaf theory, together with the trivial observation that for $U$ open in $X, \mathbf{S}_{k m n}^{q} \mathrm{U}$ equals the restriction of $S_{k m n} X$ to $U$. (See [11, pp. 38-40] for details.)

For Axiom 4, let $X$ be a manifold and $U$ be open in $X$. Now an element $h \in S_{k m n}^{q}\left(\mathbf{U}_{D}\right)$ is a set map $h: U \rightarrow \mathbf{R}$ such that $W \rightarrow U \rightarrow \mathbf{R}$ is $C^{k}$ for each variation $v: W \times \Delta^{q} \rightarrow U_{d}$ of type $m, n$. (Such a variation is precisely specified by a $C^{m}$ map $v_{1}: W \rightarrow U$.) Since $h \circ v_{1}: W \rightarrow \mathbf{R}$ must be $C^{k}$ for all such $v_{1}$, including $v_{1}=$ id: $U \rightarrow U$, it follows that $h \in C^{k}(U)$, and conversely $h \in C^{k}(U)$ implies $h \in S_{k m n}^{q}\left(U_{D}\right)$ (again we need the hypothesis $k<m$ ). It follows that $\Gamma S_{k m n} \mathbf{X}_{D}=C^{k}(X)$ for all $q$. Axiom 4 now follows from the fact that the singular coboundary $d$ alternates between the zero map and the 
identity map on $S_{\text {sing }}^{*}\left(U_{d} ; \mathbf{R}\right) \supset S_{k m n}^{*}\left(U_{D}\right)$.

The homotopy invariance of $T_{k m n}$ (Axiom 1) will follow from the homotopy invariance of the unsheafified theory $H_{k m n}=H S_{k m n}^{*}$ together with some sheaf theoretic arguments. Consider the prism operator $P: S_{q}\left(X^{\prime}\right) \rightarrow S_{q+1}\left(X^{\prime} \times I\right)$ $\rightarrow S_{q+1}\left(X^{\prime} \times \mathbf{R}\right)$ and its transpose $P^{t}: S^{q+1}\left(X^{\prime} \times \mathbf{R} ; \mathbf{R}\right) \rightarrow S^{q}\left(X^{\prime} ; \mathbf{R}\right)[7, \mathrm{p}$. 45]. The prism operator is a homotopy operator between the maps $i_{j}:$ : $S_{q} X^{\prime} \rightarrow S_{q}\left(X^{\prime} \times \mathbf{R}\right)(j=0,1)$ induced by the inclusions $i_{j}: X^{\prime} \rightarrow X^{\prime} \times\{j\}$ $\subset X^{\prime} \times \mathbf{R}$. To show that $H_{k m n}$ is homotopy invariant, it suffices to show that $P^{t}$ restricts to a map from $S_{k m n}^{q+1}(\mathbf{X} \times \mathbf{R})$ to $S_{k m n}^{q}(\mathbf{X})$. Well, if $\sigma$ is a $C^{n}$ simplex of $X^{\prime}$, then $P \sigma$ is a finite sum of $C^{n}$ simplices $P_{i} \sigma$ of $X^{\prime} \times \mathbf{R}$, and if $w \mapsto \sigma_{w}$ $(w \in W)$ is a $C^{m}$ variation of $C^{n}$ simplices of $X$, then each $w \mapsto P_{i} \sigma_{w}$ is a $C^{m}$ variation of $C^{n}$ simplices of $\mathbf{X} \times \mathbf{R}$. If $h \in S_{k m n}^{q+1}(\mathbf{X} \times \mathbf{R})$, then for each $C^{m}$ variation of $C^{n}$ simplices of $\mathrm{X}, w \mapsto \sigma_{w}$, we have

$$
\left(P^{\prime} h\right)\left(\sigma_{w}\right)=\sum_{i}\left(P_{i}^{t} h\right)\left(\sigma_{w}\right)=\sum_{i} h\left(P_{i} \sigma_{w}\right)
$$

which is a $C^{k}$ function of $w$ by definition of $\mathbf{S}_{k m n}^{q+1}(\mathbf{X} \times \mathbf{R})$. It follows that $P^{t} h \in S_{k m n}^{q}(\mathrm{X})$, as claimed. Hence $H_{k m n}$ is homotopy invariant.

Now consider the homology (derived) sheaf $H \mathrm{~S}^{*}[3$, p. 25] of the cochain complex of sheaves $\mathbf{S}_{k m n}^{*}(\mathbf{X} \times \mathbf{R})$ on $X \times \mathbf{R}$. Each point $(x, r) \in X \times \mathbf{R}$ has a basis of neighborhoods of the form $U \times J$, where $U$ is open in $X$ and $J$ is an open interval in $\mathbf{R}$. Therefore, the stalk of $H \mathbf{S}^{*}$ at $(x, r)$ is the direct (injective) limit over neighborhoods $U \times J$ of $H \Gamma\left(\mathbf{S}^{*}(\mathbf{X} \times \mathbf{R}) \mid(U \times J)\right)$, which by standard properties of direct limits, presheaves, and sheaves [3, p. 25] equals inj $\lim _{U \times J} H S^{*}(U \times J)$, which equals inj $\lim _{U} H S^{*}(U)$ by the homotopy invariance of $H S_{k m n}^{*}$ just proven. It now follows from sheaf theory, using the paracompactness of $X \times \mathbf{R}$ and the fineness of each sheaf $\mathbf{S}_{k m n}(\mathbf{X}$ $\times \mathbf{R}$ ), that $T_{k m n}$ is homotopy invariant; see [11, p. 36] for details. Q.E.D.

REMARK. It may seem strange that Theorem 3 places no restriction on $n$, the degree of differentiability of the simplices. If $n<m$, there may be $C^{n}$ simplices which cannot be varied in a $C^{m}$ variation. A cochain $h \in S_{k m n}^{q}(\mathbf{X})$ may take arbitrary (i.e. "discontinuous") values on such simplices, but $T_{k m n}$ will still be a $C^{k}$ cohomology theory.

Comparison of the different $T_{k m n}$. Inspecting the definitions, one sees that there is a natural map from $S_{k m n}^{*}(\mathbf{X})$ to $S_{k^{\prime} m^{\prime} n^{\prime}}^{*}(\mathbf{X})$ whenever $k>k^{\prime}, m<m^{\prime}$, and $n<n^{\prime}$.

THEOREM. 4. If $k>k^{\prime} \geqslant 0,0 \leqslant m<m^{\prime}$, and $0<n<n^{\prime}$, there is a natural transformation of functors: $T_{k m n}^{*} \rightarrow T_{k^{\prime} m^{\prime} n^{\prime}}^{*}$. If, in addition, $k<m$ and $k^{\prime}<m^{\prime}$ (so that $T_{k m n}$ and $T_{k^{\prime} m^{\prime} n^{\prime}}$ satisfy the axioms), then the map $T_{k m n}^{0}\left(\mathbf{X}_{D}\right) \rightarrow$ $T_{k^{\prime} m^{\prime} n^{\prime}}^{0}\left(\mathbf{X}_{D}\right)$ corresponds, under the isomorphism of Axiom 4, to the inclusion $C^{k}(X) \rightarrow C^{k^{\prime}}(X)$. Under the same hypotheses, but with $k=k^{\prime}$, if $X$ is a 
manifold and $X^{\prime}$ is $X$ with the leaf topology from some nonsingular smooth foliation, then (letting $\left.\mathbf{X}=\left(X^{\prime} \rightarrow X\right)\right) T_{k m n}^{*}(\mathbf{X}) \rightarrow T_{k m^{\prime} n^{\prime}}^{*}(\mathbf{X})$ is an isomorphism.

Proof. Only the last assertion requires proof, and it follows immediately from the Uniqueness Theorem of [11, Theorem 6.5] which states that every cohomology theory $T$ (satisfying Axioms $1,2,3)$ for which $T^{q}\left(\mathbf{Y}_{D}\right)=0$ when $q>0$ (for all manifolds $Y$ ) is determined naturally on nonsingularly foliated manifolds by its values $T^{0}\left(\mathbf{Y}_{D}\right)$. Alternately, we can study the sheaves directly (as in the proof of Theorem 6.8 of [11]) and observe that the homology sheaves $H \mathbf{S}_{k m n}^{*}(\mathrm{X})$ and $H \mathbf{S}_{k m^{\prime} n^{\prime}}^{*}(\mathrm{X})$ have naturally isomorphic stalks at every point $x \in X$. Q.E.D.

REMARK. The alternate proof shows that the foliation on $X$ need not be $C^{\infty} ; C^{r}$ will suffice, if $r \geqslant \max \left(k\left(=k^{\prime}\right) m, n, m^{\prime}, n^{\prime}\right)$.

Mapping $T_{\mathrm{DR}}$ to $T_{k m n}$ by integration. Under suitable conditions on $k, m, n$, which we will now investigate, we can map $T_{\mathrm{DR}}$ to $T_{k m n}$ by integrating smooth forms over differentiable simplices. Let $\eta \in A^{q} X$ be a smooth $\left(C^{\infty}\right)$ differential $q$-form on $X$, and let $i: X^{\prime} \rightarrow X$ be a $C^{\infty}$ map. If $\sigma: \Delta^{q} \rightarrow X^{\prime}$ is a $C^{1}$ simplex then we can integrate $\sigma^{*} i^{*} \eta$ over $\Delta^{q}$, or as is usually said, we can integrate $i^{*} \eta$ over $\sigma$. This yields a cochain $I(\eta)$ on the $C^{1}$ simplices of $X^{\prime}$. Now let $v: W \times \Delta^{q} \rightarrow X^{\prime}$ (or $w \mapsto \sigma_{w}$ ) be a $C^{m}$ variation of $C^{1}$ simplices of $\mathbf{X}$. If $m>1$, then evaluating $I(\eta)$ on $\sigma_{w}$ is the same as pulling back $\eta$ from $X$ to $W \times \Delta^{q}$ via (iv)* and then integrating (iv)* $\eta$ over $\{w\} \times \Delta^{q}$. This shows that the map $W \rightarrow \mathbf{R}$ defined by $w \mapsto I(\eta)\left(\sigma_{w}\right)$ is $C^{m}$, if $m \geqslant 1$. Thus we have shown that $I(\eta) \in S_{m m 1}^{q}(\mathbf{X})$ for any $m \geqslant 1$. Combining this result with Theorem 4 above and the definition of $T_{\mathrm{DR}}$, we get

TheOREM 5. Let $\mathrm{X} \in \mathcal{C}_{2}^{\infty}$. If $0 \leqslant k, 1 \leqslant m, 1 \leqslant n$, and $k \leqslant m$, then integration of $C^{\infty}$ forms (on $X$ ) over $C^{n}$ simplices of $X^{\prime}$ induces a natural map

$$
I: T_{\mathrm{DR}}^{*}(\mathbf{X}) \rightarrow T_{k m n}^{*}(\mathbf{X}) \text {. }
$$

ReMark. An easy application of the Uniqueness Theorem of [11] (as in the proof of Theorem 4 above) shows that $I$ is an isomorphism if $k=m=\infty$ and $\mathbf{X}$ is a nonsingularly foliated manifold.

The obstruction to mapping $T_{\mathrm{DR}}$ to $T_{\mathrm{c}}$. By Theorems 4 and 5 there are always maps

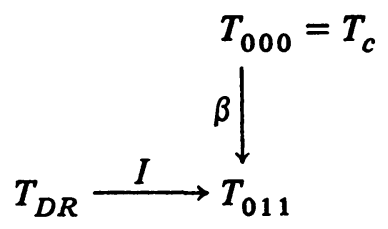

Therefore, the obstruction to mapping $T_{\mathrm{DR}}(\mathbf{X})$ to $T_{\mathrm{c}}(\mathbf{X})$ is the map $\beta$; if $I$ lifts (for example, if $\beta$ is an isomorphism) then the obstruction vanishes. 
THEOREM 6. If $\mathbf{X}$ is a (nonsingularly) foliated manifold, there is no obstruction to mapping $T_{\mathrm{DR}}(\mathbf{X})$ to $T_{\mathrm{c}}(\mathbf{X})$ (compatibly with evaluation of cocycles on cycles).

Proof. In this case, $\beta: T_{000}(\mathbf{X}) \rightarrow T_{011}(\mathbf{X})$ is an isomorphism, by Theorem 4. Q.E.D.

Now the example in the Introduction showed that Theorem 6 can be false for singularly foliated manifolds (and probably for other $\mathbf{X}$ in $\bigodot_{2}^{\infty}$ as well). Therefore the map $\beta$ cannot always be an isomorphism. To see why, factor $\beta$

$$
T_{c}=T_{000} \stackrel{\beta_{1}}{\rightarrow} T_{001} \stackrel{\beta_{2}}{\rightarrow} T_{011} .
$$

Now $\beta_{1}$ is analogous to the map $H_{\text {sing }}^{*}(M) \rightarrow H_{\mathrm{sm}}^{*}(M)$ (see Introduction); it arises from restricting cochains to smooth simplices. I do not know if $\beta_{1}$ is always an isomorphism (it is, of course, on nonsingularly foliated manifolds, by Theorem 4). Hu's theorem [9] that the inclusion $S_{\mathrm{sm}}\left(M^{\prime}\right) \rightarrow S_{\text {sing }}\left(M^{\prime}\right)$ of chains based on smooth simplices into chains based on singular (i.e. continuous) simplices is a chain homotopy equivalence for any manifold $M^{\prime}$ does not help us, because his chain inverse and chain homotopies do not necessarily vary continuously as a simplex is moved continuously in $M^{\prime}$, let alone in $M$ (where $\left.\left(M^{\prime} \rightarrow M\right) \in \mathcal{C}_{2}^{\infty}\right)$.

I find $\beta_{2}$ more interesting, because it has no analogue in ordinary cohomology. It is induced on the cochain level by including those cochains which are continuous with respect to all continuous variations of $C^{1}$ simplices into the vector space of cochains which are continuous with respect to $C^{1}$ variations of $C^{1}$ simplices. To see that $\beta_{2}$ is a nontrivial obstruction to lifting $I$, let us study the example of the Introduction and of [11, §8].

Recall that in the example, $X=\mathrm{R}^{2}=\mathrm{C}$, and $X^{\prime}=X$ topologized as $\left(\mathbb{I I}_{r} S_{r}\right) \amalg\{0\}$, where $S_{r}=\{|z|=r\}$. Consider the variation $v: \mathbf{R} \times \Delta^{1} \rightarrow \mathbf{C}$ defined by

$$
v(r, t)= \begin{cases}r \exp \left(2 \pi i t / r^{3}\right), & r \neq 0, \\ 0, & r=0 ;\end{cases}
$$

we will denote this also by $r \mapsto \sigma_{r} . v$ is a $C^{0}$ (but not $C^{1}$ ) variation of $C^{\infty}$ 1 -simplices of $\mathbf{X}$; it wraps the simplex $\{r\} \times \Delta^{1}$ around the circle $S_{|r|}$ (via $\sigma_{r}$ ) $1 / r^{3}$ times. Now consider the differential form $\eta=x d y-y d x=r^{2} d \theta$ on $X$. Integrating $\eta$ over $C^{1}$ simplices of $X^{\prime}$ gives a cochain (in fact cocycle, since $i^{*} \eta \in A^{1} X^{\prime}$ is a cocycle) $h=I(\eta) \in S_{011}^{1}(\mathbf{X})$ (by Theorem 5). Now if $h$ were continuous with respect to all $C^{0}$ variations (i.e. if $h \in S_{001}^{1}(\mathbf{X})$ ), then $r \mapsto$ $h\left(\sigma_{r}\right)$ would be a continuous function: $\mathbf{R} \rightarrow \mathbf{R}$. But $h\left(\sigma_{0}\right)=0$, while $h\left(\sigma_{r}\right)=$ $r^{2}\left(2 \pi / r^{3}\right)=2 \pi / r$, so that $\lim _{r \rightarrow 0} h\left(\sigma_{r}\right) \neq h\left(\sigma_{0}\right)$. Therefore $h$ is not an element of $S_{001}^{1}(X)$. Thus $\beta_{2}$ is an obstruction to lifting $I$ at the cochain level.

To see that $\beta_{2}$ is an obstruction at the cohomology level, too, suppose the contrary, let $h$ be as in the preceding discussion, and choose a cocycle 
$H \in S_{001}^{1}(\mathbf{X})$ so that $\beta_{2}(H)=h$ in $T_{001}^{1}(\mathbf{X})$. (Because of the sheafification in the definition of $T_{k m n}$, we may have to restrict to some neighborhood of 0 in C to do this.) Now $H$ and $h$ restrict to cohomologous singular cocycles on each $S_{r}$, so they take the same values on cycles. In particular, using the same variation $r \mapsto \sigma_{r}$, as before, but looking only at values of $r$ that make $\sigma_{r}$ a cycle (namely $r=0$ or $r=p^{-1 / 3}, p$ an integer), we see that (for such $r$ ) $H\left(\sigma_{r}\right)=h\left(\sigma_{r}\right)=2 \pi / r, r \neq 0$, while $H\left(\sigma_{0}\right)=h\left(\sigma_{0}\right)=0$, so that $r \mapsto H\left(\sigma_{r}\right)$ is discontinuous at $r=0$ as before. Thus $H \notin S_{001}^{1}(\mathbf{X})$ (contradiction). Therefore $\beta_{2}: T_{001}^{1}(X) \rightarrow T_{011}^{1}(X)$ is not an isomorphism, and moreover, $\beta_{2}$ is an obstruction to lifting the map $I$ (since $h=I(\eta)$ ).

Discussion: Choosing the "correct" continuous cohomology theory. By Theorem 3 any theory $T_{0 m n}(m \geqslant 0, n \geqslant 0)$ satisfies the axioms of a continuous cohomology theory, and by Theorem 4 all these theories agree on nonsingularly foliated manifolds. The preceding discussion shows that the various theories may differ on singularly foliated manifolds. Now it seems desirable that one be able to use differential forms when studying continuous cohomology, but to do so we must be able to map $T_{\mathrm{DR}}$ to $T_{0 m n}$ via integration of forms. We can always do this on nonsingularly foliated manifolds $\mathbf{X}$ (by Theorem 6), but in the general case the map is defined only if $m>1$ and $n \geqslant 1$. It therefore seems to me that one should choose $T_{011}$ or $T_{0 \infty \infty \infty}$ rather than $T_{\mathrm{c}}=T_{000}$ as the "correct" continuous cohomology theory to use, especially when studying Bott and Haefliger's conjectures about the continuous cohomology of $\left(B \Gamma_{q} \rightarrow B J_{q}\right)$, since it not clear whether $\beta: T_{c} \rightarrow$ $T_{011}$ is an isomorphism on this space, and hence whether $T_{\mathrm{DR}} \rightarrow T_{\mathrm{c}}$ is defined here. (The meanings of $T_{\mathrm{DR}}\left(B \Gamma_{q} \rightarrow B J_{q}\right)$, etc. will be explained in the next section.)

3. Extension of $T_{\mathrm{DR}}$ and $T_{k m n}$ to $\left(B \Gamma_{q} \rightarrow B J_{q}\right)$ and other pairs of differentiable spaces. For some purposes the category $\varrho_{2}^{\infty}$ used in the previous section is too restrictive. For example, one would like to be able to define $T_{\mathrm{DR}}$ and $T_{k m n}$ of such spaces as $\left(\Gamma_{q} \rightarrow J_{q}\right)$ and $\left(B \Gamma_{q} \rightarrow B J_{q}\right)$, where $\Gamma_{q}$ is Haefliger's smooth category of germs of diffeomorphisms of $\mathbf{R}^{q}$, with the sheaf topology [8, p. 136], $J_{q}$ is the category of $\infty$-jets of local diffeomorphisms of $\mathbf{R}^{q}$, with the $C^{\infty}$ topology [1], and $B(\cdot)$ is one of the classifying space functors on topological categories. Of course, one can already define the continuous cohomology theory $T_{\mathrm{c}}$ on these spaces (see [11, pp. 28 and 71]), but since $\Gamma_{q}$ and $J_{q}$ have a kind of smooth structure it seems reasonable to hope that we can bring to bear the techniques of differential forms on the study of the continuous cohomology of $\left(B \Gamma_{q} \rightarrow B J_{q}\right)$. Indeed, Bott, Shulman, and Stasheff have already used differential forms on $\Gamma_{q}$ and $m_{p}\left(\Gamma_{q}\right)$ (the space of composable $p$-tuples of elements of $\left.\Gamma_{q}\right)$ to study the map $H^{*}\left(B G L_{q}\right) \rightarrow H^{*}\left(B \Gamma_{q}\right)$ in ordinary cohomology ([2], [13"]). For our purposes, it will be advantageous to 
be able to define smooth functions and differential forms directly on the classifying spaces $B \Gamma_{q}, B J_{q}$, etc., instead of working only with forms on $\Gamma_{q}, m_{p} \Gamma_{q}$, etc. To do this, we shall use generalizations of manifolds called differentiable spaces.

Note. After defining differential forms on classifying spaces and geometric realizations by the differentiable space approach, I discovered the work of $\mathrm{J}$. Dupont [5'], who defined a form on the geometric realization $\|X\|$ of a semisimplicial manifold $X$ to be a collection of differential forms $\omega_{p} \in A^{*}\left(X_{p}\right.$ $\times \Delta^{p}$ ) which are compatible with respect to the face maps. One could use Dupont's forms to define $T_{\mathrm{DR}}^{*}\left(B \Gamma_{q} \rightarrow B J_{q}\right)$, but to define $T_{k m n}\left(B \Gamma_{q} \rightarrow B J_{q}\right)$ one needs a differentiable space structure in order to define smooth maps $f$ : $M \rightarrow B \Gamma_{q}$ (and smooth simplices). Another advantage of differentiable spaces for our purposes is what they allow one to define maps like $f^{*}: A^{*}\left(B \Gamma_{q}\right) \rightarrow$ $A^{*}(M)$ on the level of differential forms; in Dupont's approach this map is defined only at the cohomology level.

The concept of differentiable space, which is implicit in the calculus of variations, has been developed and utilized by J. Wolfgang Smith [14] and K. $T$. Chen [5, §1], among others. The definition of differentiable space given here is more or less a hybrid of Smith's and Chen's. In a separate paper [12] I have developed its properties, especially with regard to classifying spaces and geometric realizations of simplicial spaces; as a result I have omitted some details in the present discussion.

Definimon. A differentiable space is a topological space $X$ together with a sheaf $\mathbf{F}$ of germs of continuous real-valued functions on $X$ satisfying the closure property

$$
\begin{aligned}
& \text { If } U \text { is open in } X, f_{1}, \ldots, f_{n} \in \mathbf{F}(U)(=(\operatorname{def}) \Gamma(\mathbf{F} \mid U)) \text {, and } \\
& g \in C^{\infty}\left(\mathbf{R}^{n}\right)\left(n \text { is arbitrary), then } g \circ\left(f_{1}, \ldots, f_{n}\right) \in \mathbf{F}(U)\right. \text {. }
\end{aligned}
$$

The elements of $\mathbf{F}(U)$ (also written $C^{\infty}(U)$ ) are called smooth functions on $U$.

EXAMPLES. (1). Manifolds are differentiable spaces in an obvious way; so are $\Delta^{q}, \Delta^{q} \times I$, etc. (via restriction of smooth functions from $\mathbf{R}^{q}$, resp. $\left.\mathbf{R}^{q} \times \mathbf{R}\right)$.

(2) Every topological space $X$ is a differentiable space in a trivial way: just define $C^{\infty}(U)=C(U)$.

(3) $\Gamma_{q}$ has a locally Euclidean smooth structure generated by the coordinate functions $x_{1}, \ldots, x_{q}$ on $\mathbf{R}^{q}$, pulled back to $\Gamma_{q}$ via the source map ([0], [8]).

(4) $J_{q}$ has smooth functions corresponding to taking the source, target, and various partial derivatives of a jet; in this way $J_{q}$ is embedded in $\mathbf{R}^{\infty}=$ $\prod_{i=1}^{\infty} \mathbf{R}$. 
(5) $B G, B \Gamma_{q}$, and $B J_{q}$ ( $G$ a Lie group) are differentiable spaces if we use the Milnor-Buffet-Lor ([10], [4]) classifying space construction for a topological groupoid (= small category with inverses) $\mathcal{C}$. We recall that a point in $B \mathcal{C}$ is specified by a collection $\left\{t_{i}, g_{i j}\right\}$ satisfying

(1) $t_{i} \geqslant 0, i=0,1, \ldots$, and $t_{i}=0$ for all but a finite number of $i$.

(2) $\Sigma t_{i}=1$.

(3) $g_{i j} \in \mathcal{C}$, and is defined only if $t_{i} t_{j} \neq 0$ ( $g_{i i}$ is an identity in $\left.\mathcal{C}\right)$.

(4) $g_{i j} \circ g_{j k}=g_{i k}$ on $\left\{t_{i} t_{j} t_{k} \neq 0\right\}$.

The space $B C$ is endowed with either the weak topology, obtained as a quotient of $\amalg \Delta_{\sigma} \times m_{n} \mathcal{C}$, where $\Delta_{\sigma}$ is an $n$-face of the infinite Euclidean simplex $\Delta^{\infty}$, and $m_{n} \mathcal{C}=\{n$-tuples of composable morphisms in $\mathcal{C}\}$, or with the strong topology, which is the coarsest topology (fewest open sets) making all the $t_{i}$ and $g_{i j}$ continuous. In [12] we show (using a smooth partition of unity) that these two topologies are homotopy equivalent in a natural and smooth way.

We define the smooth structure on $B \mathcal{C}$ to be the one generated by the $t_{i}$ and $g_{i j}$. More precisely, any continuous function on $B C$ which is locally (in the strong or weak topology) a smooth function of finitely many $t_{i}$ and $g_{i j}$ is called smooth.

Now a morphism of differentiable spaces is just a continuous map which pulls back smooth functions to smooth functions.

EXAMPLe 6. $A$ smooth simplex of $X$ is just a morphism $q: \Delta^{q} \rightarrow X$ of differentiable spaces.

EXAMPLE 7. A $C^{\infty}$ foliation on a manifold $M$, when regarded as a $\Gamma_{q}$-structure with smooth transition functions, is classified by a morphism of differentiable spaces $f: M \rightarrow B \Gamma_{q}$, if we modify the usual construction of $f$ ([4], [0], J. Stasheff's Appendix C) by using a smooth partition of unity. In detail, let the $\Gamma_{q}$-structure be defined by the open cover $\left\{U_{i}\right\}$ of $M$ ( $i=$ $0,1,2, \ldots)$ and the cocycle $\left\{\gamma_{i j}: U_{i j} \rightarrow \Gamma_{q}\right\}$. Choose a subordinate smooth partition of unity $u_{i}$. Define $f$ by

$$
f(x)=\left\{t_{i}=u_{i}(x), g_{i j}=\gamma_{i j}(x)\right\} .
$$

Clearly $f$ pulls back smooth functions to smooth functions, so it is a (smooth) morphism.

If $f_{0}, \ldots, f_{n}$ are smooth functions on a differentiable space, then the symbol $f_{0} d f_{1} \wedge \cdots \wedge d f_{n}$ is identified with the collection of (smooth) differential forms $\left(f_{0} \circ g\right) d\left(f_{1} \circ g\right) \wedge \cdots \wedge d\left(f_{n} \circ g\right) \in A^{n} E$, where $E$ is an open subspace of some Euclidean space and $g: E \rightarrow X$ is smooth. The sheaf on $X$ generated by locally finite sums of symbols $f_{0} d f_{1} \wedge \cdots \wedge d f_{n}$ is denoted $\mathrm{A}^{n} X$ and called the sheaf of $n$-forms on $X$. For $U$ open in $X$, let $A^{n}(U)=\Gamma\left(\mathrm{A}^{n} X \mid U\right)$. Observe that $A^{n}(U)$ is a module over $A^{0}(U)=C^{\infty}(U)$. 
One defines

$$
H_{\mathrm{DR}}^{*}(X)=H A^{*}(X)
$$

Let $\mathscr{D}$ be the category of differentiable spaces, and let $\mathscr{D}_{2}$ be the category whose objects are morphisms $\mathbf{X}=\left(X^{\prime} \rightarrow X\right)$ of differentiable spaces. (Thus $\mathscr{D}_{2}$ contains $C_{2}^{\infty}$.) We extend $T_{\mathrm{DR}}$ to $\mathscr{D}_{2}$ by

$$
T_{\mathrm{DR}}(\mathrm{X})=H\left(\operatorname{image}\left(A^{*}(X) \rightarrow A^{*}\left(X^{\prime}\right)\right)\right)
$$

(the same formula used to define $T_{\mathrm{DR}}$ on $\mathrm{C}_{2}^{\infty}$ ).

To extend $T_{k m n}$ to $\mathscr{D}_{2}$, we first define a function on a differentiable space $X$ to be $C^{k}(k=1,2, \ldots)$ if it is locally a $C^{k}$ function of finitely many smooth functions. A continuous map $f: X \rightarrow Y$ between differentiable spaces is called $C^{k}$ if it pulls back $C^{k}$ functions to $C^{k}$ functions. ( $C^{0}$ functions and maps are defined, as usual, in terms of the given topology on $X$ rather than by the preceding definition.) We now define $C^{n}$ simplices, $C^{m}$ variations, and $C^{k}$ cochains just as we did already on $C_{2}^{\infty}$. The only tricky point is that in defining $C^{m}$ variations we still want to restrict the parameter space $W$ of the variation to be a smooth manifold if $m \geqslant 1$, or at worst a manifold with boundary or corners (if $m=0, W$ can be any space, as before). The reason we do not generalize the construction to allow $W$ to be any differentiable space is that by doing so we would have to allow the trivial cases where $W$ is any space and all continuous functions are called smooth (Example 2 above). If we did this, then every $C^{0}$ variation would be a $C^{m}$ variation and vice versa, obliterating a distinction we want to preserve.

The definitions of $S_{k m n}^{q}(\mathbf{X}), \mathbf{S}_{k m n}^{q}(\mathbf{X})$, and $T_{k m n}^{q}(\mathbf{X})$ for $\mathbf{X} \in \mathscr{D}_{2}$ now proceed as before (and coincide with the old definitions when $\mathbf{X} \in \bigodot_{2}^{\infty}$ ).

We ask next under what restrictions on $X^{\prime}$ or on $X$ the theories $T_{k m n}$ and $T_{\mathrm{DR}}$ satisfy the axioms for $C^{k}$ (resp. $C^{\infty}$ ) cohomology theories. We begin with a lemma giving a simple sufficient criterion for a certain important condition on $X$ to hold.

LEMMA 1. Let $X$ be a differentiable space. Suppose that $C^{\infty}(X)$ contains a countable set of functions $f_{1}, f_{2}, \ldots$ which separates the points of $X$, and suppose that $X$ has the topology generated by the sets $f_{i}^{-1}(V), V$ open in $\mathbf{R}$. Then $X$ is paracompact, and every open cover of $X$ admits a subordinate smooth partition of unity.

Proof. See [12].

COROllary. $J_{q}, B G$, and $B J_{q}$ (the latter two in the strong topology) are paracompact and admit smooth partitions of unity subordinate to any open cover.

Proof. See [12].

THEOREM 7. Both $T_{\mathrm{DR}}$ and $T_{k m n}(k \leqslant m)$ satisfy the axioms for a $C^{\infty}$ (resp. 
$\left.C^{k}\right)$ cohomology theory on the full subcategory of $\mathscr{D}_{2}$ containing all $\left(X^{\prime} \rightarrow X\right)$ for which $X$ is paracompact and admits smooth partitions of unity subordinate to any open cover.

Remark. In Axioms 1, 2, and 3 we allow $X^{\prime}$ and $X$ to be differentiable spaces: but in Axiom 4 (normalization) we still restrict $X$ to be a manifold, for reasons related to the point made above about variations.

Proof of theOREM. Under the hypotheses the sheaf $C^{\infty} X$ of smooth functions on $X$ is fine [3, p. 109]. Since $S_{k m n}^{q} \mathbf{X}, \mathbf{A}^{q} X$, and $A^{q} X^{\prime}$ are modules over $C^{\infty} X$, as before, Axioms 2 and 3 now follow for both theories exactly as before. Axiom 4 pertains only to manifolds, so it is already proven. The proof of Axiom 1 (homotopy invariance) for $T_{k m n}$ carries over verbatim from Theorem 3. The homotopy invariance proof for $T_{\mathrm{DR}}$ consists of showing that the same homotopy operator used on $C_{2}^{\infty}$ (see [11, p. 46]) still makes sense on $\mathscr{D}_{2}$; see [12] for details. Q.E.D.

COROLLARY. Both $T_{\mathrm{DR}}$ and $T_{k m n}$ satisfy the axioms on a category containing $\left(B \Gamma_{q} \rightarrow B J_{q}\right)$.

We remark that Theorem 5 carries over to the present context. In particular, if $m \geqslant 1$ and $n \geqslant 1$ there exist maps

$$
T_{\mathrm{DR}}\left(B \Gamma_{q} \rightarrow B J_{q}\right) \stackrel{I}{\rightarrow} T_{0 m n}\left(B \Gamma_{q} \rightarrow B J_{q}\right) \stackrel{\beta}{\leftarrow} T_{\mathrm{c}}\left(B \Gamma_{q} \rightarrow B J_{q}\right) .
$$

I do not know if $\beta$ is an isomorphism here. (This is a reason for using $T_{0 m n}$ as a "correct" continuous cohomology theory.) Finally, we can replace the complex of forms

$$
\operatorname{image}\left(A^{*} B J_{q} \rightarrow A^{*} B \Gamma_{q}\right) \quad(q \text { fixed })
$$

on $\left(B \Gamma_{q} \rightarrow B J_{q}\right)$ by the double complex of forms

$$
\bigoplus_{p, r} \operatorname{image}\left(A^{r}\left(m_{p} J_{q}\right) \rightarrow A^{r}\left(m_{p} \Gamma_{q}\right)\right) \quad(q \text { fixed })
$$

on the nerve (see [13]) of the "category with two topologies" $\left(\Gamma_{q} \rightarrow J_{q}\right)$ :

$$
\left(\mathbf{R}^{q} \rightarrow \mathbf{R}^{q}\right) \leftarrow\left(\Gamma_{q} \rightarrow J_{q}\right) \stackrel{\leftarrow}{\leftarrow}\left(m_{2} \Gamma_{q} \rightarrow m_{2} J_{q}\right) \ldots
$$

The proof that both complexes yield the same cohomology $\left(T_{\mathrm{DR}}^{*}\left(B \Gamma_{q} \rightarrow\right.\right.$ $\left.B J_{q}\right)$ ) uses the methods of [11, \$7] and is given in [12] (see also Theorem 8 below).

Discussion. The above double complex, under the name $A_{\infty}^{*}\left(\Gamma^{(*)}\right)$, appeared in work by R. Bott, H. Shulman, J. Stasheff, and S. Halperin in [13']. Bott and Haefliger (unpublished; sketched in [1]) have shown that its cohomology equals the relative Gel'fand-Fuks cohomology $H_{\mathrm{GF}}^{*}\left(a_{q}, O_{q}\right)$. Thus their original conjecture on $T_{c}^{*}\left(B \Gamma_{q} \rightarrow B J_{q}\right)$ (see Introduction above) could be proved by showing that $I$ and $\beta$ above are isomorphisms for $\left(B \Gamma_{q} \rightarrow B J_{q}\right)$. As 
we have seen, $I$ and $\beta$ are not isomorphisms in general. In the special case $\left(B\left(G_{d}\right) \rightarrow B G\right)$, however, ( $G$ a Lie group, $G_{d}=G$ with discrete topology), $I$ and $\beta$ are isomorphisms, since $T_{\mathrm{DR}}\left(B\left(G_{d}\right) \rightarrow B G\right)$ is the (van Est) smooth cohomology of $G$ (coefficients in R), while $T_{0 m n}$ and $T_{\mathrm{c}}$ equal the continuous cohomology of $G$ (see [11, pp. 72-73] and Theorem 8 below); these two cohomologies are equal by a result of G. D. Mostow [10', Theorem 3.4.1]. Hence if $I$ and $\beta$ are isomorphisms for $\left(B \Gamma_{q} \rightarrow B J_{q}\right)$, it is probably for combinatorial rather than topological reasons.

We now study the cohomologies of arbitrary classifying spaces and geometric realizations in $\mathscr{D}_{2}$ (see [13], [11, Appendix A] for background). Recall ([15], [11, Appendix A]) that the unwound geometric realization $\mu(X)$ of a (semi)simplicial space $X$ (called the Milnor realization by Tom Dieck, but differing from Milnor's original realization $|X|$ of a semisimplicial set) is defined by

$$
\mu(X)=\|X \times Z\|=\coprod_{n, \sigma} X_{n} \times \Delta_{\sigma} / \sim
$$

where $\sigma$ is an ordered (by $<)(n+1)$-tuple of nonnegative integers (all different), $\Delta_{\sigma}$ is the (nondegenerate) $n$-face of $\Delta^{\infty}$, the infinite Euclidean simplex, having $\sigma$ as its set of vertices, $X_{n}=X[n]$ (from the definition of semisimplicial space), and $\sim$ denotes the relations

$$
(x, t) \sim\left(\partial_{i} x, t\right), \quad x \in X_{n}, t \in \Delta_{\partial_{i} \sigma},
$$

where $\partial_{i}$ is the $i$ th face map. For example, if $G$ is a topological group and $N G$ its nerve [13] then $\mu(N G)=B G$, the Milnor classifying space of $G$ (in the sense of [10]). Similarly, $\mu\left(N \Gamma_{q}\right)=B \Gamma_{q}$ and $\mu\left(N J_{q}\right)=B J_{q}$ (where $B(\cdot)$ is the Milnor-Buffet-Lor construction described above, and $N\left({ }^{\cdot}\right)$ is the nerve of a topological category [13]; $N \Gamma_{q}[n]=m_{n} \Gamma_{q}$ ).

As with $B C$, there are two topologies on $\mu(X)$, denoted weak and strong, but they are smoothly homotopy equivalent [12].

If $X$ is a simplicial object in $\mathscr{D}$, then $\mu(X)$ has a natural structure of differentiable space [12], in either the strong or the weak topology. Thus if $\mathbf{X}$ is a simplicial object in $\mathscr{D}_{2}$, then $\mu(\mathbf{X})=(\operatorname{def})\left(\mu\left(X^{\prime}\right) \rightarrow \mu(X)\right) \in \mathscr{D}_{2}$.

THEOREM 8. Let $\mathbf{X}$ be a simplicial object in $\mathscr{D}_{2}$. Then there is a natural isomorphism

$$
T_{\mathrm{DR}}^{*}(\mu(\mathbf{X})) \simeq H_{\text {total }}\left(\bigoplus_{p, r} \operatorname{image}\left(A^{r}\left(X_{p}\right) \rightarrow A^{r}\left(X_{p}^{\prime}\right)\right)\right),
$$

where the total coboundary $d \pm \delta$ is formed from the exterior derivative $d$ (on $\left.A^{*}\right)$ and the simplicial coboundary $\delta=\Sigma(-1)^{i} \partial_{i}^{*}$.

The analogous statement for $T_{k m n}^{*}$, namely

$$
T_{k m n}^{*}(\mu(\mathbf{X})) \cong H_{\text {total }}\left(\bigoplus_{p, r} \Gamma \mathbf{S}_{k m n}^{r} \mathbf{X}_{p}\right)
$$


is true if $\mu(X)$ is given the strong topology and if $X_{p}$ and $\mu(X)$ are paracompact and admit smooth partitions of unity, which is the case, for example, if each $X_{p}$ satisfies the hypotheses of Lemma 1 above.

Proof. See [12].

EXAMPLE 8. $X^{\prime}=N \Gamma_{q}, X=N J_{q}, X_{p}^{\prime}=m_{p} \Gamma_{q}, X_{p}=m_{p} J_{q}, \mu(\mathbf{X})=\left(B \Gamma_{q} \rightarrow\right.$ $B J_{q}$ ). (This example was discussed above.)

ExAMPle 9. $X^{\prime}=N\left(G_{d}\right), X=N G, \mu(X)=B\left(G_{D}\right)$. (Here $G_{d}=$ Lie group $G$ with the discrete topology, and $\mathbf{G}_{D}=\left(G_{d} \rightarrow G\right)$.) We compute:

$$
\begin{aligned}
T_{\mathrm{DR}}^{*}\left(B \mathbf{G}_{D}\right) & =H_{\text {total }}\left(\bigoplus_{p, r} \operatorname{image}\left(A^{r}\left(G^{p}\right) \rightarrow A^{r}\left(G_{d}^{p}\right)\right)\right) \\
& =H\left(\bigoplus_{p} C^{\infty}\left(G^{p}\right)\right)=(\operatorname{def}) H_{\text {van Est }}^{*}(G ; \mathbf{R})
\end{aligned}
$$

in the sense of van Est's group cohomology based on $C^{\infty}$ cochains [16']. A simple argument using spectral sequences shows that $T_{k m n}^{*}\left(B \mathbf{G}_{D}\right)$ is the $C^{k}$ group cohomology of $G$. In particular, $T_{0 m n}^{*}\left(B G_{D}\right)$ is the continuous cohomology of $G$, which by $\left[1^{\prime}\right]$ equals $H_{\text {van Est }}^{*}(G)$, so that

$$
T_{\mathrm{DR}}^{*}\left(B \mathrm{G}_{D}\right)=T_{0 m n}^{*}\left(B \mathrm{G}_{D}\right)=T_{\mathrm{c}}^{*}\left(B \mathrm{G}_{D}\right)=H_{\mathrm{van} \text { Est }}^{*}(G ; \mathbf{R}) \text {. }
$$

4. Characteristic classes of foliations. We end by showing how the cohomology theories $T_{\mathrm{DR}}, T_{k m n}$, and $T_{\mathrm{c}}$ relate to characteristic classes of foliations of codimension $q$, and in particular to the variation of characteristic classes. We begin by observing that there are homomorphisms

$$
\begin{aligned}
& \varphi_{\mathrm{DR}}: T_{\mathrm{DR}}^{*}\left(B \Gamma_{q} \rightarrow B J_{q}\right) \rightarrow H_{\text {sing }}^{*}\left(B \Gamma_{q} ; \mathbf{R}\right), \\
& \varphi_{k m n}: T_{k m n}^{*}\left(B \Gamma_{q} \rightarrow B J_{q}\right) \rightarrow H^{*}\left(B \Gamma_{q}\right), \\
& \varphi_{\mathrm{c}}: T_{\mathrm{c}}^{*}\left(B \Gamma_{q} \rightarrow B J_{q}\right) \rightarrow H^{*}\left(B \Gamma_{q}\right) .
\end{aligned}
$$

The construction of these homomorphisms is not completely straightforward, since $T_{k m n}$ and $T_{\mathrm{c}}$ are sheafified theories. We therefore prove more general results now.

TheOREM 9. Let $\left(i: X^{\prime} \rightarrow X\right) \in \mathcal{C}_{2}$ (i.e. $i: X^{\prime} \rightarrow X$ is a continuous map between arbitrary topological spaces). Then there is a natural homomorphism

$$
\varphi_{\mathrm{c}}: T_{\mathrm{c}}^{*}\left(X^{\prime} \rightarrow X\right) \rightarrow H_{\text {sing }}^{*}\left(X^{\prime} ; \mathbf{R}\right)
$$

induced by evaluation on homology classes of $X^{\prime}$.

REMARK. If we use the unsheafified theory $H_{\mathrm{c}}$ instead of $T_{\mathrm{c}}$ (see $\left.\$ 1\right)$, then, as observed by Bott and Haefliger [1], a map $H_{\mathrm{c}}^{*}\left(X^{\prime} \rightarrow X\right) \rightarrow H_{\text {sing }}^{*}\left(X^{\prime}\right)$ is defined directly via the inclusion $S_{c}^{*}\left(X^{\prime} \rightarrow X\right) \subset S^{*}\left(X^{\prime}\right)$. In the sheafified case, we always have a map

$$
T_{c}^{*}\left(X^{\prime} \rightarrow X\right) \rightarrow T_{c}^{*}\left(X^{\prime} \rightarrow X^{\prime}\right)=(\operatorname{def}) T_{c}^{*}\left(X^{\prime}\right) .
$$

If $X^{\prime}$ is paracompact and has the homotopy type of a simplicial complex, 
then $T_{\mathrm{c}}^{*}\left(X^{\prime}\right)=H_{\text {sing }}^{*}\left(X^{\prime} ; \mathbf{R}\right)$ by [11, Theorem 2.6], so that Theorem 9 is easy in this case. The value of Theorem 9 is that it is valid even for "bad" spaces like $X^{\prime}=B \Gamma_{q}$ (which is not paracompact).

ProOF OF TheOREM (inspired by Bott [0, pp. 70-71]). Observe first that $H^{*}\left(X^{\prime} ; \mathbf{R}\right)=\operatorname{Hom}_{\mathbf{R}}\left(H_{*}\left(X^{\prime} ; \mathbf{R}\right), \mathbf{R}\right)$ so that it suffices to show how to evaluate any $\alpha \in T_{\mathrm{c}}^{q}\left(X^{\prime} \rightarrow X\right)$ on any $b \in H_{q}\left(X^{\prime}\right)$. Represent $b$ by a triple $\left(P, b_{1}, f\right)$, where $P$ is a finite simplicial complex (a geometric realization of $b$ ), $b_{1} \in H_{q}(P), f: P \rightarrow X^{\prime}$, and $f_{*}\left(b_{1}\right)=b$. Pull back $\alpha$ to $f^{*} \alpha \in T_{c}^{*}(P)$ via the morphism $(f$, if $): P=(P \rightarrow P) \rightarrow\left(i: X^{\prime} \rightarrow X\right)$. By [11, Theorem 2.6], $T_{\mathrm{c}}^{*}(P)$ $=H_{\text {sing }}^{*}(P ; \mathbf{R})$, so that we may evaluate $f^{*} \alpha$ on $b_{1}$ to get a real number we shall denote $\langle\alpha, b\rangle$. An argument similar to the one in Bott [0, pp. 70-71] shows that this number is independent of the choices made. Q.E.D.

THEOREM 10. Let $\left(i: X^{\prime} \rightarrow X\right) \in \mathscr{D}_{2}$. There are natural homomorphisms

$$
\begin{aligned}
& \varphi_{\mathrm{DR}}: T_{\mathrm{DR}}^{*}\left(X^{\prime} \rightarrow X\right) \rightarrow H_{\mathrm{sm}}^{*}\left(X^{\prime} ; \mathbf{R}\right), \\
& \varphi_{k m n}: T_{k m n}^{*}\left(X^{\prime} \rightarrow X\right) \rightarrow H_{\mathrm{sm}}^{*}\left(X^{\prime} ; \mathbf{R}\right)
\end{aligned}
$$

(where $H_{\mathrm{sm}}$ denotes singular cohomology based on smooth simplices).

Proof. Modify the proof of Theorem 9. Since we work with smooth simplices, the map $f: P \rightarrow X^{\prime}$ will be a morphism of differentiable spaces. The rest is straightforward. Q.E.D.

ReMark. The map $H_{\text {sing }}^{*}\left(X^{\prime}\right) \rightarrow H_{\mathrm{sm}}^{*}\left(X^{\prime}\right)$ is an isomorphism if $X^{\prime}$ is a manifold, or more generally, if $X^{\prime}$ is locally Euclidean; this is essentially because one can compute homology using simplices small with respect to a given open cover of $X^{\prime}$. In particular, it is an isomorphism for $X^{\prime}=\Gamma_{q}$ (this was observed by Bott, Shulman, and Stasheff [2]), and for $X^{\prime}=B \Gamma_{q}$ (again by comparing $H_{\text {sing }}$ and $H_{\mathrm{sm}}$ on an open cover and using the extended Mayer-Vietoris Theorem; see [11, pp. 14 and 71] and [12] for more details).

Now since the elements of $H^{*}\left(B \Gamma_{q} ; \mathbf{R}\right)$ are in one-to-one correspondence with characteristic classes of codimension $q$ foliations [0, pp. 69-71], we see that the elements of $T_{\mathrm{DR}}^{*}, T_{k m n}$, and $T_{\mathrm{c}}^{*}\left(B \Gamma_{q} \rightarrow B J_{q}\right)$ can be regarded as characteristic classes of foliations. Indeed, Bott and Haefliger [unpub.] have shown that $T_{\mathrm{DR}}^{*}\left(B \Gamma_{q} \rightarrow B J_{q}\right)=H_{\mathrm{GF}}^{*}\left(\mathfrak{a}_{q}, O_{q}\right)=H^{*}\left(W O_{q}\right)$, which contains the characteristic classes constructed geometrically (i.e. by connections and curvatures) by Bott, Haefliger, Kamber, Tondeur, Godbillon, Vey, et al. It seems quite conceivable that $T_{k m n}^{*}\left(B \Gamma_{q} \rightarrow B J_{q}\right)$ contains other characteristic classes for foliations. We investigate now what properties these have if they exist.

We have seen (see Example 7 above) that a codimension $q$ foliation on $M$ can be classified by a (smooth) morphism $f: M \rightarrow B \Gamma_{q}$. This induces a homomorphism 


$$
T_{k m n}^{*}(f): T_{k m n}^{*}\left(B \Gamma_{q} \rightarrow B J_{q}\right) \rightarrow T_{k m n}^{*}(M)=H^{*}(M ; \mathbf{R})
$$

whose image contains the $T_{k m n}$ characteristic classes of the foliation. Now it is not hard to see that a (smooth) one-parameter family of codimension $q$ foliations on $M$ (that is, a codimension $q+1$ foliation on $M \times \mathbf{R}$ restricting to a codimension $q$ foliation on each $M \times\{t\})$ can be classified by a commutative diagram of smooth maps

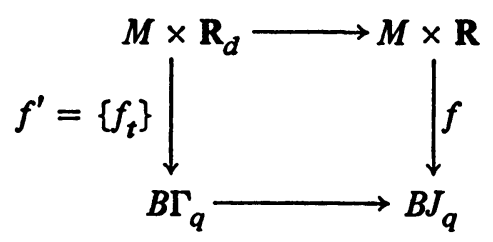

or in the language of two topologies, by a morphism

$$
\text { f: } M \times \mathbf{R}_{D} \rightarrow\left(B \Gamma_{q} \rightarrow B J_{q}\right)
$$

in $\mathscr{D}_{2}$. (Here $\mathbf{R}_{d}=\mathbf{R}$ with the discrete topology, and $\mathbf{R}_{D}=\left(\mathbf{R}_{d} \rightarrow \mathbf{R}\right)$.) Now $\mathbf{f}$ induces homomorphisms

$$
\begin{aligned}
T_{\mathrm{DR}}(\mathbf{f}): T_{\mathrm{DR}}^{*}\left(B \Gamma_{q} \rightarrow B J_{q}\right) & \rightarrow T_{\mathrm{DR}}^{*}\left(M \times \mathbf{R}_{D}\right)=\operatorname{Hom}_{\mathrm{R}}\left(H_{*}(M: \mathbf{R}), C^{\infty}(\mathbf{R})\right), \\
T_{k m n}(\mathbf{f}): T_{k m n}^{*}\left(B \Gamma_{q} \rightarrow B J_{q}\right) & \rightarrow T_{k m n}^{*}\left(M \times \mathbf{R}_{D}\right)=\operatorname{Hom}\left(H_{*}(M), C^{k}(\mathbf{R})\right), \\
T_{\mathrm{c}}(\mathbf{f}): T_{\mathrm{c}}^{*}\left(B \Gamma_{q} \rightarrow B J_{q}\right) & \rightarrow T_{\mathrm{c}}^{*}\left(M \times \mathbf{R}_{D}\right)=\operatorname{Hom}\left(H_{*}(M), C^{0}(\mathbf{R})\right)
\end{aligned}
$$

(the right-hand equalities follow from [11, p. 92]). In other words, a class $\alpha \in T_{k m n}\left(B \Gamma_{q} \rightarrow B J_{q}\right)$, when pulled back to a foliated manifold $M$, varies in a $C^{k}$ manner as the foliation is varied smoothly. Similarly, classes in $T_{\mathrm{c}}\left(B \Gamma_{q}\right.$ $\left.\rightarrow B J_{q}\right)$ vary continuously, while classes in $T_{\mathrm{DR}}\left(B \Gamma_{q} \rightarrow B J_{q}\right)$ vary smoothly. (Of course, since $T_{\mathrm{DR}}\left(B \Gamma_{q} \rightarrow B J_{q}\right)=H^{*}\left(W O_{q}\right)$ the last fact can be seen by observing that any of the functorial constructions of characteristic classes in terms of connections and curvatures (such as Kamber and Tondeur's [ $\left.{ }^{\prime}\right]$ ] depends smoothly on parameters; in this connection, see also Heitsch [ $\left.8^{\prime}\right]$.)

By results we have obtained in this paper, the map

$$
\varphi_{\mathrm{DR}}: T_{\mathrm{DR}}^{*}\left(B \Gamma_{q} \rightarrow B J_{q}\right) \rightarrow H_{\text {sing }}^{*}\left(B \Gamma_{q} ; \mathbf{R}\right)
$$

factors through $\varphi_{k m n}$ for any $k \geqslant 0$ provided $m, n>1$. Also, $\varphi_{k m n}$ factors through $\varphi_{k^{\prime} m^{\prime} n^{\prime}}$ provided $k>k^{\prime}>0,0<m<m^{\prime}$, and $0<n<n^{\prime}$. In particular, $\varphi_{c}$ factors through any $\varphi_{0 m n}$. We see then that all of the maps factor through $\varphi_{0 \infty \infty \infty}$. In particular, $T_{0 \infty \infty}^{*}\left(B \Gamma_{q} \rightarrow B J_{q}\right)$ is a promising place to look for new characteristic classes for foliations. The elements of $T_{0 \infty \infty \infty}\left(B \Gamma_{q}\right.$ $\rightarrow B J_{q}$ ) include all the known classes which can be constructed from connection and curvatures (namely $H^{*}\left(W O_{q}\right)$ ) but may also include new classes which do not vary smoothly as a foliation is varied smoothly. 
We end with some open problems.

(1) Does this approach in fact yield new characteristic classes for foliations? That is, is the image of $T_{0 \infty \infty \infty}\left(B \Gamma_{q} \rightarrow B J_{q}\right)$ in $H^{*}\left(B \Gamma_{q} ; \mathbf{R}\right)$ strictly larger than image $T_{\mathrm{DR}}\left(B \Gamma_{q} \rightarrow B J_{q}\right)=$ image $H^{*}\left(W O_{q}\right)$ ? If Bott and Haefliger's conjecture that $T_{\mathrm{DR}}\left(B \Gamma_{q} \rightarrow B J_{q}\right)=T_{\mathrm{c}}\left(B \Gamma_{q} \rightarrow B J_{q}\right)$ is correct, then probably $T_{000 \infty}$ $=T_{\mathrm{c}}\left(B \Gamma_{q} \rightarrow B J_{q}\right)$, so that we would not find any classes this way. In this case, however, we would have the curious result that the classes in $T_{\mathrm{c}}$, which $a$ priori vary only continuously, must in fact vary smoothly when a foliation is varied smoothly.

(2) If $\alpha$ is a characteristic class for foliations which varies in a $C^{k}$ manner as a foliation is varied smoothly, does it follow that $\alpha \in$ image $T_{k m n}\left(B \Gamma_{q} \rightarrow\right.$ $B J_{q}$ ) for some $m, n$ ? (We have seen that the converse is true.) If $\alpha$ varies smoothly, must it come from $T_{\mathrm{DR}}^{*}\left(B \Gamma_{q} \rightarrow B J_{q}\right)$ (i.e. from $\left.H^{*}\left(W O_{q}\right)\right)$ ?

(3) Try to find general relations between the image of $T_{k m n}^{p}\left(X^{\prime} \rightarrow X\right)$ in $H^{p}\left(X^{\prime} ; \mathbf{R}\right)$ and the vector space of $C^{k}$ homomorphisms from $H_{p}\left(X^{\prime}\right)$ to $\mathbf{R}$. (One must first find a good definition of the latter vector space.) The examples computed in [11] (for the $C^{0}$ and $C^{\infty}$ cases, but easily modified to the $C^{k}$ case) should be useful for testing possible relationships. Any progress on this problem would shed light on problem 2 above.

\section{REFERENCES}

0. R. Bott, Lectures on characteristic classes and foliations, Lecture Notes in Math., vol. 279, Springer-Verlag, Berlin and New York, 1972, pp. 1-94.

1. , Some remarks on continuous cohomology, Proc. Internat. Conf. on Manifolds and Related Topics in Topology, Tokyo, 1973, pp. 161-170. MR 51 \# 4269.

2. R. Bott, H. Shulman and J. Stasheff, De Rham theory for classifying spaces, Advances in Math. 20 (1976), 43-56.

3. G. E. Bredon, Sheaf theory, McGraw-Hill, New York, 1967. MR 36 \#4552.

4. J.-P. Buffet and J.-C. Lor, Une construction d'un universel pour une classe assez large de r-structures, C. R. Acad. Sci. Paris Sér. A 270 (1970), A640-A642. MR 42 \#6823.

5. K. T. Chen, Iterated integrals of differential forms and loop space homology, Ann. of Math. (2) 97 (1973), 217-246. MR 52 \# 1756.

5'. J. L. Dupont, Simplicial de Rham cohomology and characteristic classes of flat bundles, Aarhus Universitet Preprint series, 1974-75, No. 29; also Topology 15 (1976), 233-245.

6. S. Eilenberg, Singular homology in differentiable manifolds, Ann. of Math. (2) 48 (1947), 670-681. MR 9, 52.

7. M. Greenberg, Lectures on algebraic topology, Benjamin, New York and Amsterdam, 1967. MR 35 \#6137.

8. A. Haefliger, Homotopy and integrability, Manifolds-Amsterdam 1970, Lecture Notes in Math., vol. 197, Springer-Verlag, Berlin and New York, 1971, pp. 133-163. MR 44 \#2251.

8'. J. L. Heitsch, Deformations of secondary characteristic classes, Topology 12 (1973), 381-388. MR 47 \#9639.

9. S.-T. Hu, On singular homology in differentiable spaces, Ann. of Math. (2) 50 (1949), 266-269. MR 10, 728.

9. F. Kamber and P. Tondeur, Characteristic invariants of foliated bundles, Manuscripta Math. 11 (1974), 51-89. MR 48 \#12556. 
10. J. Milnor, Construction of universal bundles. II, Ann. of Math. (2) 63 (1956), 430-436. MR $17,1120$.

10. G. D. Mostow, Cohomology of topological groups and solvmanifolds, Ann. of Math. (2) 73 (1961), 20-48. MR 23 \#A2484.

11. M. A. Mostow, Continuous cohomology of spaces with two topologies, Mem. Amer. Math. Soc. No. 175, 1976. MR 54 \# 1253.

12. _ Differential geometry on Milnor classifying spaces and geometric realizations, $\mathbf{J}$. Differential Geometry (to appear).

13. G. B. Segal, Classifying spaces and spectral sequences, Inst. Hautes Ėtudes Sci. Publ. Math. No. 34 (1968), 105-112. MR 38 \#718.

13'. H. Shulman, The double complex of $\Gamma_{k}$, Proc. Sympos. Pure Math., vol. 27, Amer. Math. Soc., Providence, R. I., 1975, pp. 313-314. MR 51 \#11504.

13". H. Shulman and J. Stasheff, De Rham theory for BГ, Proc. Conf. on Foliations, Rio de Janeiro, January 1976.

14. J. Wolfgang Smith, The de Rham theorem for general spaces, Tôhoku Math. J. (2) 18 (1966), 115-137. MR 34 \#2027.

15. T. tom Dieck, On the homotopy type of classifying spaces, Manuscripta Math. 11 (1974), 41-49. MR 50 \#3222.

16. I. Vaisman, Cohomology and differential forms, Dekker, New York, 1973. MR 49 \#6095.

16'. W. T. van Est, Group cohomology and Lie algebra cohomology in Lie groups. I, II, Nederl. Akad. Wetensch. Proc. Ser. A 56 = Indag. Math. 15 (1953), 484-504. MR 15, 505.

17. F. W. Warner, Foundations of differentiable manifolds and Lie groups, Scott, Foresman, Glenview, III., 1971. MR 45 \#4312.

Department of Mathematics, University of Illinois at Urbana-Champaign, Urbana, ILLNNOIS 61801

Current address: Department of Mathematics, North Carolina State University, Raleigh, North Carolina 27607 